hep-ph/0204216

April, 2002

\title{
Effects of initial axion production and photon-axion oscillation on type Ia supernova dimming
}

\author{
Yuval Grossman*, ${ }^{1}$ Sourov Roy ${ }^{\dagger},{ }^{1}$ and Jure Zupan ${ }^{\ddagger 2}$ \\ ${ }^{1}$ Department of Physics, Technion-Israel Institute of Technology \\ Technion City, 32000 Haifa, Israel \\ ${ }^{2}$ Josef Stefan Institute \\ Jamova 39, P.O.Box 3000, SI-1001 Ljubljana, Slovenia
}

\begin{abstract}
Recently, Csáki, Kaloper and Terning (CKT) suggested that axion-photon oscillation in the intergalactic medium can explain the observed dimming of distant type Ia supernovae. This mechanism works only if the initial axion flux is much smaller than the initial photon flux. We study several mechanisms for such initial axion production. The direct axion production in the supernovae, the photon-axion oscillation in the magnetic field of supernovae and in the magnetic field of their host galaxies are addressed. We find it likely that the initial axion flux is very small and therefore does not pose a problem to the CKT mechanism.
\end{abstract}

\footnotetext{
*yuvalg@physics.technion.ac.il

$\dagger$ roy@physics.technion.ac.il

$\ddagger$ jure.zupan@ijs.si
} 


\section{INTRODUCTION}

Type Ia Supernovae (SNe Ia) are very interesting objects in observational cosmology [1]. Recent observations of distant $(z \sim 1)$ SNe Ia have raised this interest enormously because the data indicate that they are fainter than would be expected for a decelerating universe [2, 3]. The dimming seems to be independent of the wave length of the emitted light. The standard interpretation of this result is that our universe is accelerating at present as a result of a non-vanishing cosmological constant. Several alternatives to this idea have been suggested. One idea suggests that the light from distant SNe Ia can be partially absorbed by the dust which may be present on the light path [4]. Ideas involving extra dimensions have also been suggested [5]. The effect of cosmic evolution on distant SNe Ia was studied in [6].

Recently, Csáki, Kaloper and Terning (CKT) [0] proposed another explanation of the dimming due to oscillation of photons into very light axions in the background magnetic field of the intergalactic plasma. The statistical significance of this result and possible generalizations of the mechanism are discussed in Ref. [8].

The intergalactic plasma produces an energy dependent photon-axion oscillation and thus a frequency dependent dimming [9, 10, 11]. This is in contrast to the achromatic dimming caused by a non-vanishing cosmological constant. While in the future the energy dependence may be the best tool to discriminate between these two mechanicsms, the present data are not sensitive enough [10, 11]. Based on this fact, it is important to further investigate the CKT mechanism.

In all these works [7, 8, 9, 10, 11] it was implicitly assumed that only photons are emitted from the supernova (SN) while axions are produced only via oscillation in the intergalactic medium. If this assumption failed the dimming would be less significant. As an example consider the case of polarized photons, where the initial axion flux is equal to the initial photon flux (with polarization state parallel to the external magnetic field). Then, since the photon to axion and axion to photon oscillation probabilities are equal $\left(P_{\gamma \rightarrow a}=P_{a \rightarrow \gamma}\right)$ the oscillation will cause no effect at all. More generally, in the case of unpolarized photons and/or randomly distributed background magnetic field, the photon intensity on Earth is given by

$$
I_{\gamma}(y)=I_{\gamma}(0)-P_{\gamma \rightarrow a}(y)\left[I_{\gamma}(0)-2 I_{a}(0)\right]
$$


where $y$ is the distance traveled by the photons, $I_{\gamma}(0)\left[I_{a}(0)\right]$ is the initial photon [axion] flux, $I_{\gamma}(y)$ is the final photon flux and $P_{\gamma \rightarrow a}(y)$ is the oscillation probability. Clearly, if the initial axion flux is significant it must be taken into account.

In this paper we estimate the initial axion flux for the relevant energy range. We consider axion production in the $\mathrm{SN}$, axion conversion in the $\mathrm{SN}$ magnetic field and axion conversion in the magnetic field of the host galaxy. We find that it is unlikely that the initial axion flux is significant. We thus conclude that neglecting the initial axion flux does not pose a problem to the axion-photon mixing explanation of the dimming.

\section{PHOTON-AXION MIXING FORMALISM}

In a background magnetic field axions and photons oscillate into each other [12, 13] due to the axion-photon coupling

$$
\mathcal{L}_{\text {int }}=\frac{a}{M} \vec{E} \cdot \vec{B},
$$

where $a$ is the axion field, $\vec{E}$ is the electric field of the photon, $\vec{B}$ is the background magnetic field and the mass scale $M$ characterizes the strength of the axion-photon interactions. In Ref. [7] it was found that $M=4 \times 10^{11} \mathrm{GeV}$ gives the best fit to the data and we will use this value in the analysis. In order to have mixing a transverse external magnetic field is needed. Moreover, only the plane wave photon polarization state parallel to the external field can mix with the axion.

Photon-axion oscillation is induced by the following mass-squared matrix

$$
\mathcal{M}^{2}=\left(\begin{array}{cc}
\omega_{p}^{2} & i \mathcal{E} \mu \\
-i \mathcal{E} \mu & m^{2}
\end{array}\right) .
$$

where $\mathcal{E}$ is the photon energy,

$$
\mu=\frac{B}{M},
$$

$B \sim|\mathbf{B}|$ is the projection of the magnetic field on the direction of the photon polarization and $m$ is the axion mass. In Ref. [7], the authors found that $m \sim 10^{-16} \mathrm{eV}$ is needed. For the purposes of this paper, as long as $m^{2} \ll \max \left(\mathcal{E} \mu, \omega_{p}^{2}\right)$, we do not need the exact value of the axion mass ( $m \sim 10^{-16} \mathrm{eV}$ is small enough). The plasma frequency is given by

$$
\omega_{p}^{2}=\frac{4 \pi \alpha n_{e}}{m_{e}},
$$


where $m_{e}$ is the electron mass and $n_{e}$ is the number density of electrons in the plasma. We omitted the Euler-Heisenberg term [14 $\omega_{E H}^{2}=(7 \alpha / 90 \pi)\left(B / B_{c r}\right)^{2} \mathcal{E}^{2}$ (where $B_{c r}=4.41 \times$ $10^{13} \mathrm{G}$ is the critical field strength [13]), which contribute to $\mathcal{M}_{11}^{2}$, since the magnetic fields we are considering are much smaller than $B_{c r}$ and the effect of this term is negligible.

For a constant magnetic field the oscillation probability of photons into axions is given by

$$
P_{1}=\frac{\mu^{2}}{k^{2}} \sin ^{2}\left(\frac{k x}{2}\right)=\frac{(\mu x)^{2}}{4}\left(\frac{\sin (k x / 2)}{k x / 2}\right)^{2},
$$

where $x$ is the distance traveled by the photon, $k=2 \pi / L_{O s c}$ and the oscillation length is given by

$$
L_{O s c}=\frac{4 \pi \mathcal{E}}{\sqrt{\left(\omega_{p}^{2}-m^{2}\right)^{2}+4 \mu^{2} \mathcal{E}^{2}}} .
$$

Note that the oscillation probability is bounded by

$$
P_{1} \leq \frac{(\mu x)^{2}}{4} .
$$

Of particular interest to us is the case where the constant magnetic field domain size $\left(L_{d o m}\right)$ is small, namely when $k L_{d o m} \ll 1$. Expanding in this small parameter one finds that the bound of Eq. (8) (with $x=L_{d o m}$ ) is saturated, namely $P_{1}=\left(\mu L_{d o m}\right)^{2} / 4$. In particular, in this case the conversion is independent of the axion mass, the photon energy and the plasma frequency. This is the reason that the CKT mechanism does not produce energy dependent dimming as long as the plasma frequency is small enough such that the above approximation is valid [10].

Next we consider the case where the magnetic field is not constant. Within a set of simplifying assumptions (see the Appendix for the derivation) the total photon-axion conversion over many domains is given by

$$
P_{\gamma \leftrightarrow a}(y)=\frac{1}{3}\left(1-e^{-y / L_{\text {decay }}}\right),
$$

such that $y=N L_{d o m}$ is the total length traveled by the photons in $N$ domains and

$$
L_{\text {decay }}=\frac{2 L_{\text {dom }}}{3 P_{1}},
$$

where $P_{1}$ is given in (6). Using (8) we find the following bound

$$
P_{\gamma \leftrightarrow a}(y) \leq \frac{(y \mu)^{2}}{8 N} .
$$


As discussed in the appendix there are several assumptions that enter the derivation of (9). Still, we do not expect significant deviation from the bound (11) in more realistic cases.

We also consider cases where the mean free path of photons, $L_{m f p}$, is not very large. In such cases it takes an average of $N=3 R^{2} / L_{m f p}^{2}$ random steps to escape from a region of radius $R$. Assuming that the magnetic field is constant over a region of order $L_{m f p}$, the conversion probability is given by Eq. (9) with

$$
y=N L_{m f p}=\frac{3 R^{2}}{L_{m f p}}, \quad L_{\text {decay }}=\frac{2 L_{m f p}}{3 P_{1}},
$$

Using (11) we get the bound

$$
P_{\gamma \leftrightarrow a}(y=R) \leq \frac{3(R \mu)^{2}}{8} .
$$

Note that the bound does not depend on the photon mean free path.

In the following we will make use of the bounds (8), (11) and (13). Of course, they are useful only when the characteristic scale set by the magnetic field $(1 / \mu)$ is much larger than the relevant scales in the problem $(x, y$ and $R)$. As it turns out this is indeed the situation in the cases we study.

\section{PHOTON-AXION MIXING INSIDE SUPERNOVA IA}

We now turn to study axion-photon mixing inside the supernova and determine under which physical conditions there is an appreciable photon-axion oscillation probability. SNe Ia are identified by the variations of their apparent luminosity with time. The intrinsic

peak luminosity of these SNe varies within $20 \%$, and based on the time variation even this variation can be corrected for. This is the reason that they are used as "standard candles" for various cosmological tests. The spectral analysis of SNe Ia shows the absence of hydrogen. Supernovae of this type are believed to result from the thermonuclear explosion of White Dwarfs (WDs) when their masses are very close to the Chandrasekhar limit [15, 16].

In order to estimate the axion-photon oscillation probability inside SNe Ia we consider a simple model [17]. The supernova is assumed to be a sphere of uniform density with an initial radius $R_{0} \sim 10^{9} \mathrm{~cm}$ that expands with an outer velocity of $v=c / 30=10^{9} \mathrm{~cm} \mathrm{~s}^{-1}$. Photons are emitted uniformly throughout the volume of the supernova and the energy distribution follows Planck's law [17, 18]. The peak luminosity occurs within 10 to 20 days after the explosion. The progenitor stars are magnetized objects. Some WDs are known to 
host magnetic fields of intensity ranging between $10^{5}$ and $10^{9} \mathrm{G}$. The magnetic fields during the supernova event are not very well understood. It is possible that due to the turbulent motions prior to the explosion the WD goes through a stage which might dramatically change the intensity of its initial magnetic field. In particular, if the effect is to increase the magnetic field its value can be as large as $B_{0} \sim 10^{11} \mathrm{G}$ [19. After the explosion of the WD, the ejecta undergo a large expansion. In a homologous expansion all components of the field decrease like

$$
\frac{B}{B_{0}}=\frac{\left(R_{0}\right)^{2}}{(R)^{2}} \approx \frac{\left(R_{0}\right)^{2}}{(v t)^{2}}
$$

where the approximation is valid for $v t \gg R_{0}$. This background magnetic field can be chaotic which is a likely result of the turbulent motion prior to the explosion.

We first consider the production of axions in the SN envelope due to photon conversion in its magnetic field. The photon mean free path at peak luminosity is not well known. Ref. [18], for example, estimates that $L_{m f p}$ is in the range $10^{6}-10^{14} \mathrm{~cm}$. The SN radius at that time, which is of order $10^{15} \mathrm{~cm}$, is larger than the mean free path. The conversion probability at peak luminosity is therefore bounded by Eq. (13). Using (14), we get

$$
P_{\gamma \leftrightarrow a} \leq \frac{3}{8}\left(\frac{B_{0} R_{0}^{2}}{M v t}\right)^{2} \approx 3 \times 10^{-8}\left(\frac{B_{0}}{10^{11} \mathrm{G}}\right)^{2}\left(\frac{10 \text { days }}{t}\right)^{2}
$$

where for the last step we used $M=4 \times 10^{11} \mathrm{GeV}, R_{0}=10^{9} \mathrm{~cm}$ and $v=c / 30$. We learn that for values of $t \geq 10$ days and $B_{0} \leq 10^{11} \mathrm{G}$ the conversion probability is negligible. Our conclusions would not hold in the unlikely case that initial magnetic fields in SNe Ia reach values significantly higher than $10^{14} \mathrm{G}$.

Let us now discuss other processes by which axions could in principle be produced inside the SN. It is well known that axion production is potentially large in the first stage of type II supernovae explosions [20]. One would then like to check whether a similar situation occurs in the type Ia SNe explosions. However, this is not the case since the temperature and the density of matter in the plasma of SNe Ia at its peak luminosity are relatively very small. Let us elaborate on this.

The potentially relevant processes are the ones that occur at the typical energy scale of a few $\mathrm{eV}$. The axion luminosity due to these processes scales as $n_{B}^{p} n_{e}^{r} T^{s}$, where $n_{B}$ is the baryon density, $n_{e}$ the electron density, $T$ the temperature, and $p, r$, and $s$ are process dependent nonnegative numbers. We consider the following processes: axiorecombination - i.e. the reaction, where axions are emitted when electrons and ions form a bound state; 
axion bremsstrahlung from the electrons and nuclei in the plasma; Compton scattering with axion instead of a photon in the final state; and the axion emission from electrons via the conversion of longitudinal plasmons. The luminosity due to the axiorecombination scales as $n_{B} n_{e} T^{3 / 2}$ [21], the luminosity due to the bremsstrahlung from electrons scales as $n_{B} n_{e} T^{5 / 2}[20]$. The axion bremsstrahlung emission from nuclei in the eV energy range is suppressed by additional powers of $m_{e} / m_{N}$. The luminosity due to the Compton scattering scales as $n_{e} T^{6}$ [20], and the luminosity due to the plasmon conversion scales as $n_{e}^{2} T[22]$. The density of plasma in SNe Ia at the time of peak luminosity is of order $10^{-13} \mathrm{~g} \mathrm{~cm}^{-3}$ and the temperature is about $2 \times 10^{4} \mathrm{~K}[18]$. To get a rough estimate of the axion production

in the SNe Ia we compare these values with the typical density $\rho \sim 10^{13} \mathrm{~g} \mathrm{~cm}^{-3}$ and the temperature of several $\mathrm{MeV}$ inside the core of SNe II [20]. Multiplying by the volume of the SNe explosion remnant and taking $n_{e, B} R^{3}$ roughly constant we arrive at a suppression factor of about $10^{-40}-10^{-50}$. This expectation is borne out by a more detailed calculation, where it is found that the axion flux due to these processes in the SNe Ia remnant is suppressed by about 50 orders of magnitude compared to the photon flux. We conclude that axion production by these mechanisms can be safely neglected.

\section{PHOTON-AXION MIXING IN THE HOST GALAXY}

Next we consider photon axion conversion in the host galaxy. The details of the background magnetic field of the galaxy are not well understood. We first assume that far away galaxies are similar to the better known nearby galaxies. The size of the galactic disk, which is also the coherence length of the smooth component of the magnetic field is of order $1 \mathrm{kpc} \sim 10^{26} \mathrm{eV}^{-1}$. For a typical galaxy, Ref. [23] estimates a smooth toroidal component of the magnetic field of magnitude about $2 \mu \mathrm{G}$ and a random component with a magnitude of about $5 \mu \mathrm{G}$ and a coherence length of order $50 \mathrm{pc}$. The mean electron density in the host galaxy is taken to be $n_{e}=0.03 \mathrm{~cm}^{-3}$ [23, 24]. With these parameters we find that the mean free path of photons is much larger than $1 \mathrm{kpc}$ and thus they basically do not interact after they leave the SN. For the smooth toroidal component of magnetic field, using $B=2 \mu \mathrm{G}$, we have $\mu \sim 10^{-28} \mathrm{eV}$. Using (8) with $x=L_{\text {dom }}=1 \mathrm{kpc}$ we conclude

$$
P_{\gamma \leftrightarrow a} \lesssim 10^{-4}
$$


The random component of the magnetic field is somewhat larger in strength. However, due to smaller coherence length its contribution to the conversion probability is likely to be even smaller. For example, using (11) with $L_{\text {dom }}=50 \mathrm{pc}, y=1 \mathrm{kpc}$ and $B=5 \mu \mathrm{G}$ we find

$$
P_{\gamma \leftrightarrow a} \lesssim 10^{-5}
$$

Far away host galaxies may have considerably different magnetic field than what we find in nearby galaxies. One or two order of magnitude enhancement of the magnetic field could result in a significant conversion. (In order for such conversion to be realized also the plasma frequency has to be small.) Yet, there is no well motivated reason to believe that this is the case. Thus it is likely that the conversion probability in the far away host galaxy is small.

\section{CONCLUSIONS}

We have studied axion production in type Ia supernovae and photon-axion oscillations in their magnetic fields and in the magnetic fields of their host galaxies. If such axion production is significant it can disfavor the photon-axion oscillation explanation of the observed dimming of light from far away type Ia supernovae since then the dimming is less significant, and it may also become energy dependent. We found that all these effects are very small. Our result is robust in the sense that it does not rely on many unknown parameters of the SNe and their host galaxies. For example, we did not have to use exact values of the plasma frequency or the photon mean free path. We had to use only the rough size of the star, its temperature and its magnetic field. We therefore conclude that axion production in the supernovae and their host galaxies has a negligible effect on the CKT explanation of the supernovae dimming.

\section{Acknowledgments}

We are grateful to Borut Bajc, Adam Burrows, Arnon Dar, Yossi Nir, Yael Shadmi and Eli Waxman for helpful discussions. J.Z. thanks the Technion theory group for hospitality while this work was in progress. Y.G. was supported in part by the Israel Science Foundation under Grant No. 237/01-1 and by the United States-Israel Binational Science Foundation (BSF) through Grant No. 2000133. J.Z. was supported in part by the Ministry of Education, Science and Sport of the Republic of Slovenia. 


\section{APPENDIX A: CONVERSION IN VARYING BACKGROUND}

In this appendix we discuss photon to axion conversions in the varying magnetic field background. To simplify the discussion it is assumed that photons and axions traverse $N$ domains of equal length. In each domain the magnetic field $B$ is assumed to be homogeneous with a discrete change in $B$ from one domain to the other, i.e. the situation is far from adiabatic conditions. The component of the magnetic field perpendicular to the direction of flight is assumed to have random orientation but is equal in size in each domain. The domains can be (not necessarily) separated by an interval where the conversion is negligible.

Consider an initial state $c_{1}(0)\left|\gamma_{1}\right\rangle+c_{2}(0)\left|\gamma_{2}\right\rangle+c_{a}(0)|a\rangle$. The two photon polarization states, $\left|\gamma_{1,2}\right\rangle$, correspond respectively to photons parallel and perpendicular to the magnetic field in the first domain. The initial photon and axion fluxes are then $I_{\gamma}(0) \sim\left|c_{1}(0)\right|^{2}+$ $\left|c_{2}(0)\right|^{2}$ and $I_{a}(0) \sim\left|c_{a}(0)\right|^{2}$ respectively. In the $n$-th domain the magnetic field is tilted by an angle $\theta_{n}$ compared to the magnetic field in the first domain

$$
\left|\gamma_{\|}^{n}\right\rangle=c_{n}\left|\gamma_{1}\right\rangle+s_{n}\left|\gamma_{2}\right\rangle, \quad\left|\gamma_{\perp}^{n}\right\rangle=-s_{n}\left|\gamma_{1}\right\rangle+c_{n}\left|\gamma_{2}\right\rangle
$$

or

$$
c_{1}(y)=c_{n} c_{\|}^{n}(y)-s_{n} c_{\perp}^{n}(y), \quad c_{2}(y)=s_{n} c_{\|}^{n}(y)+c_{n} c_{\perp}^{n}(y)
$$

where $c_{n} \equiv \cos \theta_{n}$ and $s_{n} \equiv \sin \theta_{n}$. The magnetic field mixes photons polarized parallel to the magnetic field with the axions according to the mass matrix given in (3). We denote the transition elements for one domain $t_{\gamma \rightarrow \gamma}=\left\langle\gamma_{\|}^{n}\left(L_{d o m}\right) \mid \gamma_{\|}^{n}(0)\right\rangle$ and similarly for $t_{\gamma \rightarrow a}$. The values of the transition elements are equal in each domain since the magnitude of the magnetic field has been assumed to be the same everywhere. The transition probability for photon to axion oscillation in one domain is $P_{1}=\left|t_{\gamma \rightarrow a}\right|^{2}$ with $P_{1}$ given in (6), while the survival probability of photon is $1-P_{1}=\left|t_{\gamma \rightarrow \gamma}\right|^{2}$. At the end of the $n$-th domain the photon and axion fluxes are

$$
\begin{aligned}
I_{\gamma}(n+1) & \sim\left(1-P_{1} c_{n}^{2}\right)\left|c_{1}\left(y_{n}\right)\right|^{2}+\left(1-P_{1} s_{n}^{2}\right)\left|c_{2}\left(y_{n}\right)\right|^{2}+P_{1}\left|c_{a}\left(y_{n}\right)\right|^{2}+\ldots \\
I_{a}(n+1) & \sim P_{1} c_{n}^{2}\left|c_{1}\left(y_{n}\right)\right|^{2}+P_{1} s_{n}^{2}\left|c_{2}\left(y_{n}\right)\right|^{2}+\left(1-P_{1}\right)\left|c_{a}\left(y_{n}\right)\right|^{2}+\ldots
\end{aligned}
$$

where dots represent terms that are proportional to $\cos \theta_{n}, \sin \theta_{n}$ or $\cos \theta_{n} \sin \theta_{n}$. We have

defined $y_{n}=(n-1) L_{\text {dom }}$. Namely, the coefficients $c_{1,2, a}$ are taken at the beginning of the $n$-th domain. 
Next we assume that the transition probability in one domain is small i.e., $P_{1} \ll 1$, and that the direction of the magnetic field is random, i.e., $\theta_{n}$ is a random variable with $\theta_{n+1}-\theta_{n} \sim \mathcal{O}(1)$. In this limit $c_{n}^{2}$ and $s_{n}^{2}$ can be replaced by their average value $1 / 2$, while $c_{n}, s_{n}$ and $c_{n} s_{n}$ are averaged to zero. Using the fact that $I_{\gamma}(n) \sim\left|c_{1}\left(y_{n}\right)\right|^{2}+\left|c_{2}\left(y_{n}\right)\right|^{2}$ and $I_{a} \sim\left|c_{a}\left(y_{n}\right)\right|^{2}$ we arrive at

$$
\begin{aligned}
\left(\begin{array}{c}
I_{\gamma}(n+1) \\
I_{a}(n+1)
\end{array}\right)= & \left(\begin{array}{cc}
1-\frac{1}{2} P_{1} & P_{1} \\
\frac{1}{2} P_{1} & 1-P_{1}
\end{array}\right)\left(\begin{array}{c}
I_{\gamma}(n) \\
I_{a}(n)
\end{array}\right)= \\
& \left(\begin{array}{cc}
\frac{2}{3}+\frac{1}{3}\left[1-\frac{3}{2} P_{1}\right]^{n+1} & \frac{2}{3}-\frac{2}{3}\left[1-\frac{3}{2} P_{1}\right]^{n+1} \\
\frac{1}{3}-\frac{1}{3}\left[1-\frac{3}{2} P_{1}\right]^{n+1} & \frac{1}{3}+\frac{2}{3}\left[1-\frac{3}{2} P_{1}\right]^{n+1}
\end{array}\right)\left(\begin{array}{c}
I_{\gamma}(0) \\
I_{a}(0)
\end{array}\right) .
\end{aligned}
$$

We can then use the fact that the number of domains is large and replace $\left(1-3 P_{1} / 2\right)^{n+1}$ with the limiting expression $\exp \left[-\left(3 P_{1} y\right) /\left(2 L_{d o m}\right)\right]$ to arrive at the final expressions

$$
\begin{aligned}
& I_{\gamma}(y)=I_{\gamma}(0)-P_{\gamma \rightarrow a}\left(I_{\gamma}(0)-2 I_{a}(0)\right), \\
& I_{a}(y)=I_{a}(0)+P_{\gamma \rightarrow a}\left(I_{\gamma}(0)-2 I_{a}(0)\right),
\end{aligned}
$$

with

$$
P_{\gamma \rightarrow a}=\frac{1}{3}\left(1-e^{-y / L_{\text {decay }}}\right)
$$

and $L_{\text {decay }}=\left(2 L_{\text {dom }}\right) /\left(3 P_{1}\right)$. In cases where the oscillation length is much larger than the domain size the conversion probability $P_{1}$ saturates the upper bound $P_{1}=\left(\mu L_{d o m}\right)^{2} / 4$ and we arrive at the expression $L_{\text {decay }}=8 /\left(3 \mu^{2} L_{d o m}\right)$ given also in [7].

[1] For a recent review on type Ia supernovae, see e.g., B. Leibundgut, Ann. Rev. Astron. \& Astrophys. 39, 67 (2001) astro-ph/0003326].

[2] S. Perlmutter et al. [the Supernova Cosmology Project Collaboration], Astrophys. J. 517, 565 (1999) astro-ph/9812133.

[3] A.G. Riess et al. [the Supernova Search Team Collaboration], Astron. J. 116, 1009 (1998) astro-ph/9805201.

[4] A.N. Aguirre, Astrophys. J. 525, 583 (1999) astro-ph/9904319;

A.N. Aguirre and Z. Haimann, Astrophys. J. 532, 28 (2000) astro-ph/9907039. 
[5] G.R. Dvali, G. Gabadadze and M.A. Shifman, Phys. Lett. B497, 271 (2001) [hepth/0010071]; C. Deffayet, G.R. Dvali and G. Gabadadze, Phys. Rev. D 65, 044023 (2002) astro-ph/0105068.

[6] P.S. Drell, T.J. Loredo and I. Wasserman, Astrophys. J. 530, 593 (2000) astro-ph/9905027.

[7] C. Csáki, N. Kaloper, and J. Terning, Phys. Rev. Lett. 88, 161302 (2002) hep-ph/0111311.

[8] J. Erlich and C. Grojean, Phys. Rev. D 65, 123510 (2002) hep-ph/0111335.

[9] C. Deffayet, D. Harari, J-P. Uzan and M. Zaldarriaga, hep-ph/0112118.

[10] C. Csáki, N. Kaloper, and J. Terning, Phys. Lett. B535, 33 (2002) hep-ph/0112212].

[11] E. Mörtsell, L. Bergström and A. Goobar, astro-ph/0202153.

[12] P. Sikivie, Phys. Rev. Lett. 51, 1415 (1983) ; Erratum-ibid. 52, 695 (1983).

[13] G. Raffelt and L. Stodolsky, Phys. Rev. D 37, 1237 (1988) .

[14] W. Heisenberg and H. Euler, Z. Phys. 98, 714 (1936); S.L. Adler, Ann. Phys. (N.Y.) 67, 599 (1971).

[15] S. Sarkar, hep-ph/0201140.

[16] P. B. Pal, Pramana 54, 79 (2000) hep-ph/9906447.

[17] P.A. Pinto and R.G. Eastman, Astrophys. J. 530, 744 (2000).

[18] P.A. Pinto and R.G. Eastman, Astrophys. J. 530, 757 (2000).

[19] P. Ruiz-Lapuente and H.C. Spruit, Astrophys. J. 500, 360 (1998) astro-ph/9711248.

[20] G. G. Raffelt, "Stars As Laboratories For Fundamental Physics: The Astrophysics Of Neutrinos, Axions, And Other Weakly Interacting Particles," Chicago, USA: Univ. Pr. (1996).

[21] S. Dimopoulos, J.A. Frieman, B.W. Lynn and G.D. Starkman, Phys. Lett. B179, 223 (1986).

[22] N. V. Mikheev, G. Raffelt and L. A. Vassilevskaya, Phys. Rev. D 58, 055008 (1998) [hepph/9803486].

[23] E.D. Carlson, Phys. Lett. B344, 245 (1995) .

[24] D. Harari and P. Sikivie, Phys. Lett. B289, 67 (1992). 